\title{
HYBRID ENERGY HARVESTING SYSTEM BASED ON MICROFLUIDICS WITH INTERFACE CIRCUITRY
}

\author{
Arhatha Bramhanand ${ }^{l}$, Md. Mahbubur Rahman ${ }^{1}$, Young-Chul Bae ${ }^{2}$, and Hanseup Kim ${ }^{l}$ \\ ${ }^{1}$ Electrical and Computer Engineering, University of Utah, Salt Lake City, Utah, USA \\ ${ }^{2}$ Electrical, Electronic Communication and Computer Engineering, Chonnam National University, South Korea
}

\begin{abstract}
We present a fluidic-based energy harvesting device that incorporates microfluidic structures, which can hydraulically disperse and accommodate large input force into a micro structure; and power handling circuitry, which converts varying energy to support a commercial wireless link, into a single layer of a shoe insole. The microfluidic structures utilize hydraulic amplification of deflection and de-amplification of force to enhance power generation through electromagnetic induction while preventing potential mechanical fracture of the micro structures. The power regulation circuitry converts AC power into DC at each fluidic channel and then adds them into high voltage output. The fabricated energy harvesting system generated a raw power ${ }_{\text {rms }}$ output of $7.28 \mathrm{~mW}$ under $647.5 \mathrm{kPa}$ pressure and power density of $1 \mathrm{~mW} / \mathrm{cm}^{3}$ from a device volume of $7.2 \mathrm{~cm}^{3}$. The integrated power regulating circuitry demonstrated power conversion efficiency of $62.8 \%$.
\end{abstract}

\section{INTRODUCTION}

Recently wireless systems are finding immensely increasing applications in parallel to the technological advances in low power circuits and digital communication technology $[1,2]$. In order to be truly independent and portable, such wireless systems require highcapacity (long-life) and reliable power sources that can also be small in volume and light in weight. Since the minimum power, required for some sensor applications, becomes sufficiently low within few $\mathrm{mW}$ ranges, an alternative approach of micro-scale energy harvesting has been actively pursued to replace the electrochemical batteries that have been traditionally utilized but require periodic replacement and cause environmental hazards due to harmful chemical contents, such as lead, mercury and cadmium. Micro-scale energy harvesting technology harvests electrical power from ambient environments (vibration, temperature, fluid flows) and thus is permanent and environmental friendly [3-5].

Despite rigorous progress, micro-scale energy harvesting systems have typically demonstrated limited power generation, ranging from a few $\mathrm{nW}$ to $\mathrm{mW}$ with an average power density of $100 \mathrm{~W} / \mathrm{cm}^{3}$ [6,7]. Such low power outputs could stem from conventional uses of relatively weak and mild environmental energy sources due to the limited range of inputs (force and deflection) that a micro device can accommodate under its small and fragile structure. Recently some notable demonstration by producing large output power under heavy body weights $(>60 \mathrm{~kg})$ through piezoelectric materials have been performed particularly for shoes $[8,9]$ in a relatively large scale with some limitations in practicality.

In order to overcome the above issues we utilize a new class of microfluidic-based energy harvesting device that (1) can accommodate relatively high input force and large deflection into micro-scale structures through hydraulic de-amplification and (2) can provide thin and flexible structures appropriate for a shoe insole. A simple proof-of-concept on the operation principle of a microfluidic energy harvesting was previously reported $[10,11]$. Here, we report an integrated system that incorporates both (1) the power handling circuitry and the multiple-channel device.
Particularly, we investigated two different multiple-channel configurations: (2) a bundle structure that has independent and "fluidically non-connected" energy harvesting channels and (3) an all-connected channel device. These multi-channel structures allow electromagnetic amplification proportional to that of the number of channels for enhanced power generation.

\section{MICROFLUIDIC ENERGY HARVESTING SYSTEM Design}

The developed energy harvesting system comprises two major sections: two chambers with deformable elastic diaphragm on the top and fluidic channels interconnecting them (Fig. 1). The chambers and fluidic channels together form a closed system and are filled with a working fluidic medium (DI water) without air bubbles to ensure efficient hydraulic operation. Present inside the channels is a train of $\mathrm{N}$ equally sized cylindrical permanent magnets and N-1 non-magnetic spacers which are alternately placed and free to move within the channel. The movement of the magnet-spacer train is confined by ring shaped stoppers which are placed at either ends of the channel. Conductive coils are wound on the outer surface of each channel. These coils are placed at regular intervals and the spacing between coils is equal to the magnet length, to account for phase-matching.

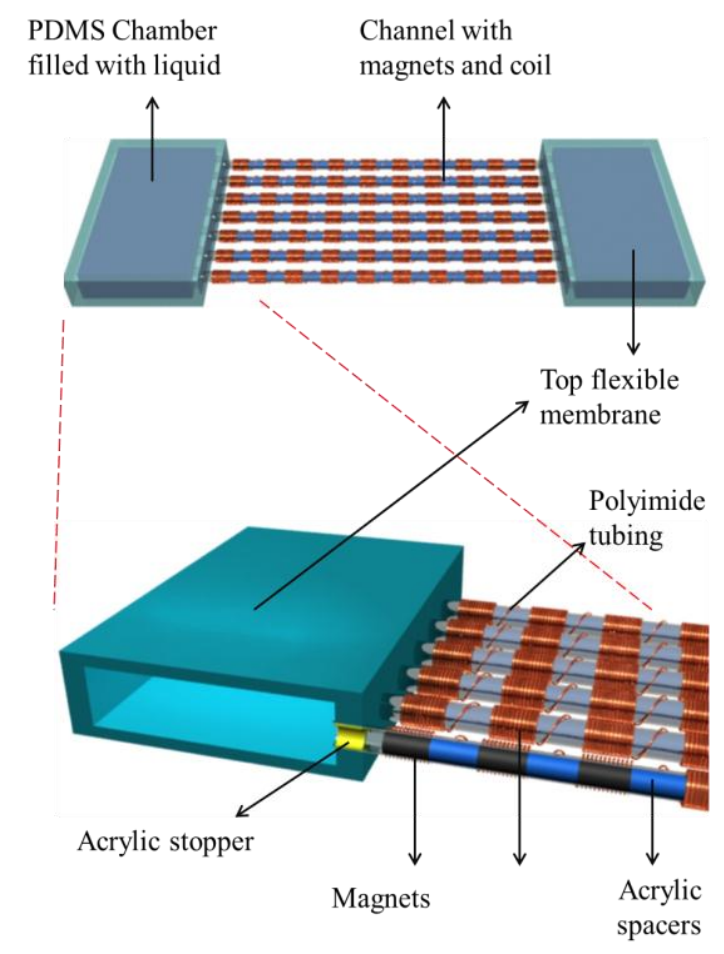

Figure 1: Illustration of the energy harvesting, fluidic based system (Top). A partial cross-section of the PDMS chamber and polyimide channel with stopper, magnets and spacers (Bottom). 


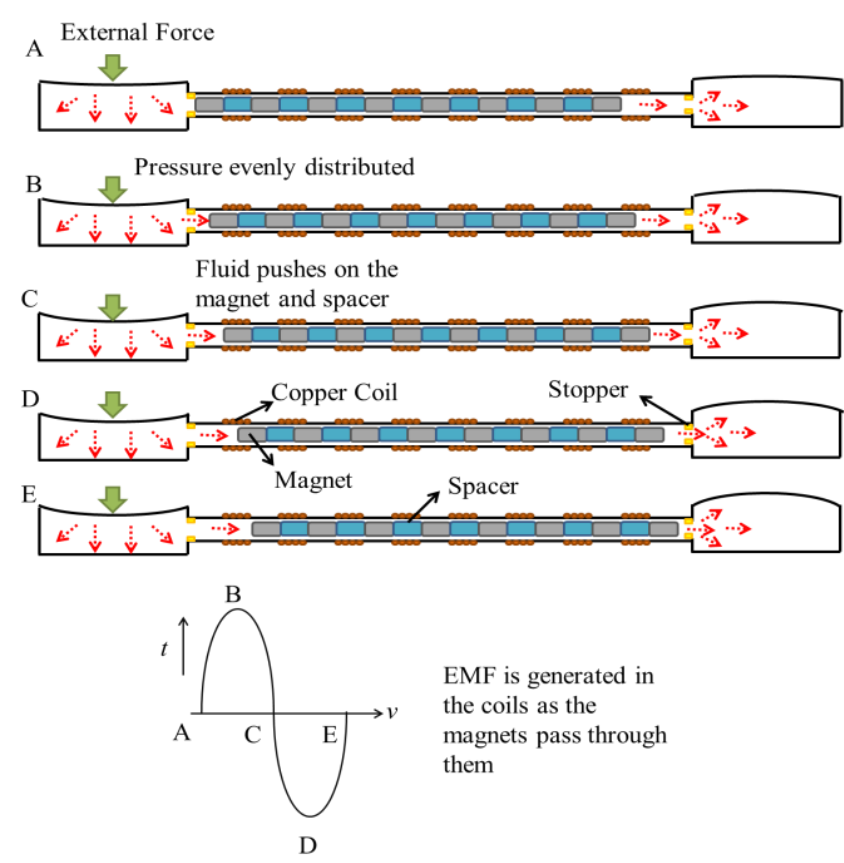

Figure 2: Operation principle of the energy harvesting system.

\section{Operation}

The device, while incorporated into the shoe insole, operates based on the applied pressure at the heel and toes where maximum pressure is available. The applied pressure is accommodated by each diaphragm at both chambers alternatively. The chambers accommodate the high deflection force and transform it into hydraulic pressure that is evenly distributed in accordance with Pascal's law. Since the hydraulic pressure is equal at all points within the chamber and the cross sectional area of the channel is much smaller than the area over which the force was exerted a multiplication of force can be realized. The hydraulic pressure pushes the magnets in the channels. As the magnets pass through the coils emf is induced in the coils and energy is generated (Fig.2). By alternately applying pressure on the two diaphragms the magnets can be continuously shuttled thru and fro in the channels, giving a continuous power output.

\section{Fabrication}

An improved fabrication process is adopted compared to previous reports [10,11] First, polyimide tubes of inner radius of $0.86 \mathrm{~mm}$ are used to form the fluidic micro channels. Inside is a train of $\mathrm{NdFeB}$ neodymium rare earth, grade $\mathrm{N} 52$, axially magnetized permanent magnets $(0.8 \mathrm{~mm}$ radius and $3.13 \mathrm{~mm}$ in length) located, while laser-cut acrylic spacers are assembled between the magnets. Second, insulated copper coils of $0.15 \mathrm{~mm}$ wire with 22turns are wound around the channel. Acrylic laser cut ring like stoppers are placed at either end of the channel. Next the deformable chambers are made of PDMS, Sylgard 184 base and curing agent are mixed in a 10:1 ratio, then poured into molds and cured at $60^{\circ} \mathrm{C}$. Then the channels are inserted into the side walls of the chamber and the two PDMS structures are bonded together using PDMS-PDMS bonding technology. Different PDMS-PDMS bonding methods were tested and the uncured PDMS bonding method proved to be the strongest which could sustain a pressure of up-to $648 \mathrm{kPa}$ for our device. After the bonding a syringe is inserted along the interface between the channel and chamber wall on one side to remove the air and completely fill the system with D.I. water. This opening is later sealed with silicone glue.

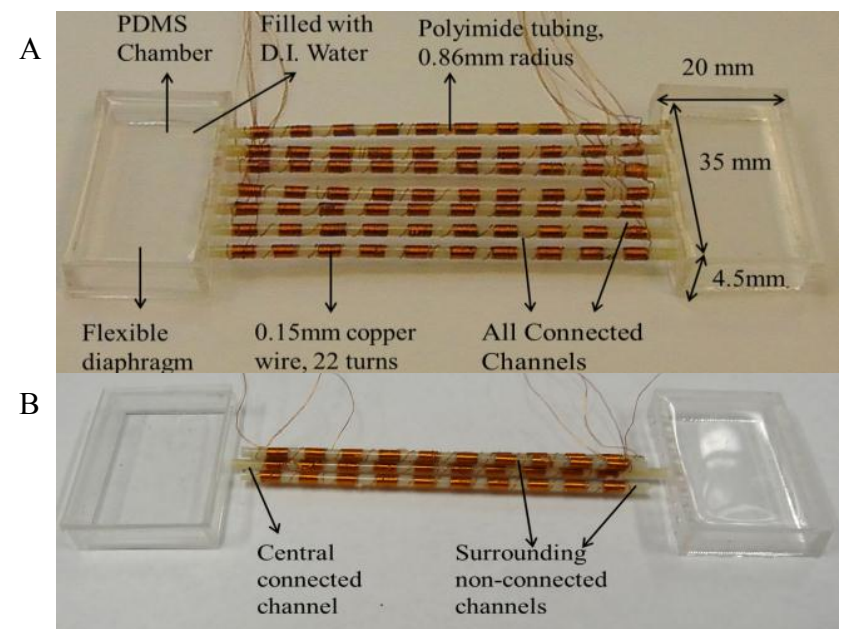

Figure 3: A: Fabricated all-connected multi-channel energy harvesting device. B: Fabricated bundle-channel energy harvesting device.

\section{Multichannel Structures}

The device performance can be improved by having multiple channels between the two chambers. This can be simply analyzed by connecting multiple channels between the chambers such that when an external force is applied the working fluid flows through all the connected channels (Fig 3-A). Thus the displaced working liquid pushes the magnets in all the channels leading to an increase in the total output. Since the increase in the device volume is not large the generated power density increases.

Another approach is the bundle system multi-channel which electromagnetically-amplifies the output for the same amount of work done in pushing the magnets along the channel. The rare earth $\mathrm{NdFeB}$ magnets have high magnetic field flux and as a result when channels are placed close to each other they show strong magnetic field interaction between the magnets in the adjacent channels. This can be used to an advantage by having one main channel connecting the two chambers and independent fludically not-connected channels which are placed around the main channel (Fig 3-B). Thus when an external force is applied the magnets in the main channel move as explained earlier. At the same time due to magnetic interactions between the main channel and surrounding channels, the magnets in the surrounding channel are pulled along. Thus by having additional independent channels higher power can be generated for the same applied pressure without reducing flow velocity or displacement in each channel.

\section{EXPERIMENTAL VALIDATION}

\section{Testing Methods}

The output response of the two multi-channel energy harvesting systems was monitored with respect to the applied external pressure under a single (impulse) actuation condition. The tested systems include an all-connected multi-channel device and a bundle-channel device. The all-connected multi-channel device has seven channels with ten magnet coil pairs in each channel, while the bundle device has one central channel and four surrounding independent channels where in each channel had ten magnet coil pair. Force was applied on one of the diaphragm and monitored by a CSD200 Chatillon dynamometer that has a detection resolution of $1 \mathrm{~N}$ in force. All the coils from each channel were connected in series so that voltage amplification due to phase matching could be obtained, and the resultant sinusoidal output was observed across an optimum load on a Tektronix TDS 2024B oscilloscope. 


\section{Results and Discussion}

Figure 4 shows the produced power output under various input pressure ranges for the fabricated all-connected multichannel device. The output power increases linearly with the increase in the external pressure applied. The device provided a maximum raw power $_{\text {rms }}$ of $7.28 \mathrm{~mW}$ for the input pressure of $647.5 \mathrm{kPa}$. This corresponded to the power density of $1 \mathrm{~mW} / \mathrm{cm}^{3}$. The maximum pressure that could be applied was determined by the maximum PDMS-PDMS bonding strength. Different bonding methods such as plasma bonding, epoxy adhesive, silicone glue, UV curable glue, partial PDMS curing, and uncured PDMS bonding were tested (Table 1) to monitor the maximum burst pressure and select the strongest bonding methodology. It is observed that the uncured PDMS-PDMS bonding method has been most successful which could sustain pressure up to $648 \mathrm{kPa}$.

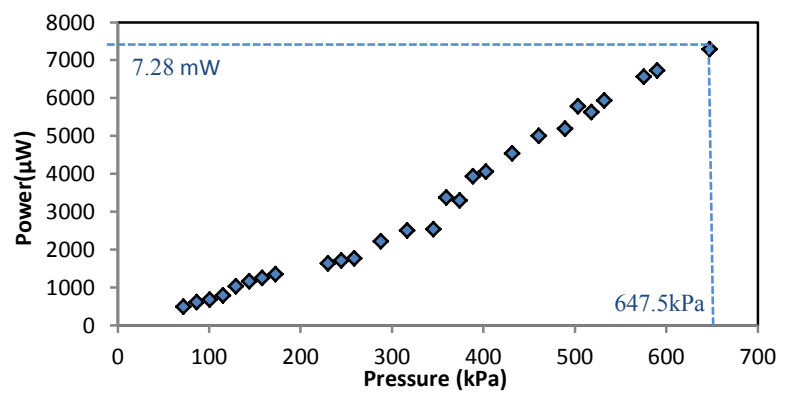

Figure 4: Power(rms) versus pressure for the all-connected multichannel device.
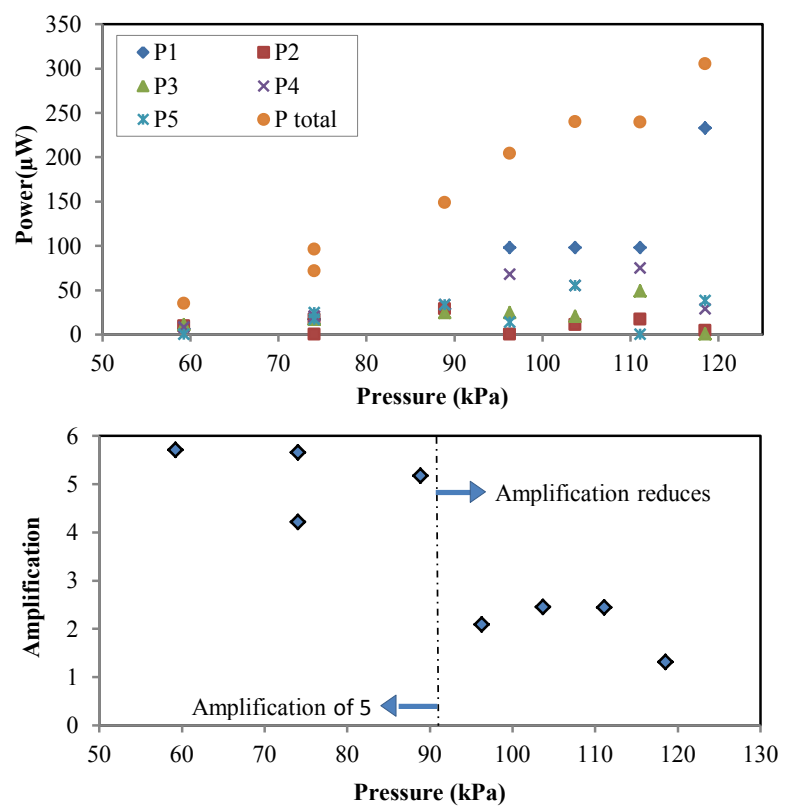

Figure 5: Pressure response obtained for bundle structure device. P1- Power generated in main channel. P2, P3, P4, P5 - Power generated in surrounding channels(top). Resulted in amplification factor proportional to number of channels up-to $90 \mathrm{kPa}$ (bottom).

Figure 5 shows the resultant power generation in a bundledchannel device with 5 channels. In the bundle system, when the magnets in the central channel move, those in the surrounding four independent channels were also induced to move through electromagnetic field. At a lower pressure range $(<90 \mathrm{kPa})$ the power output was amplified proportional to the total number of channels. However, at a higher pressure range $(>90 \mathrm{kPa})$, the amplification factor was reduced (Fig. 5). As pressure increases, the velocity of fluid flow in each channel increases, failing to move magnets in the surrounding channels. In order to enhance the advantages of this approach, optimization of the distance between the channels had to be performed such that they were not too far off which reduces the pull efficiency at higher pressure. The tested device produced a total power output of $305 \mathrm{~W}$ for an input pressure of $118 \mathrm{kPa}$; but at this pressure range an amplification of only 1.5 was observed (Fig. 5). At a lower pressure range $(<90 \mathrm{kPa})$, full amplification of 5 to 5.7 was observed. Thus, it was observed that the bundled-channel device works well only for low pressure applications.

Table 1: Maximum burst pressure for different bonding methods.

\begin{tabular}{lc}
\hline Bonding Method & Max Burst Pressure (kPa) \\
\hline Plasma bonding & unsuccessful bonding \\
Epoxy adhesive & 10 \\
Silicone glue & 254 \\
UV curable glue & 554 \\
Partial curing & 609 \\
Uncured PDMS & $\mathbf{6 4 8}$ \\
\hline
\end{tabular}

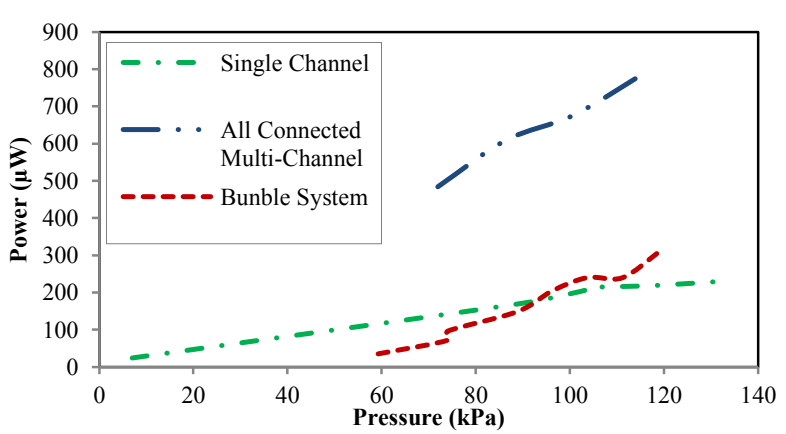

Figure 6: Comparison of the pressure response of the single channel[10], all connected multi-channel and bundle system device.
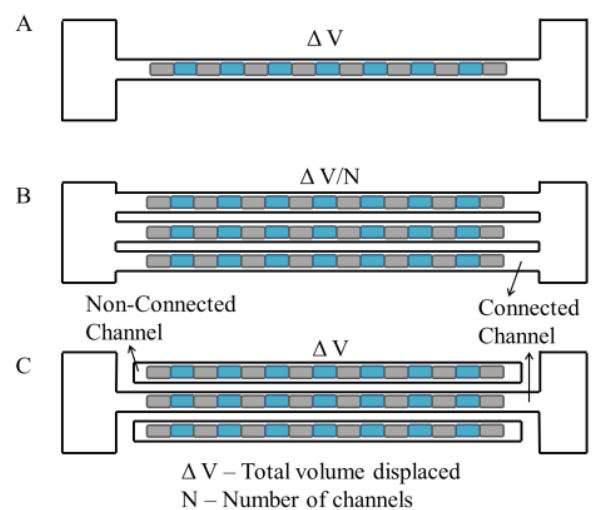

Figure 7: Distribution of displaced liquid in (A)single channel[10], (B)all connected multi-channel and (C)bundle system device.

Figure 6 compares the obtained power outputs for the previously reported single channel device [10] and the tested multi-channel devices under various input pressure ranges. In both single channel and the bundled-channel devices, the total volume of displaced liquid flows only through the central channel. However, in the bundle-channel, such flows and the resultant magnetic movement also induce the movement of magnet in the 
surrounding channel. On the other hand, the total volume of the displaced liquid is distributed through the multiple channels in the all connected multi-channel device (Fig. 7). Although the bundle system theoretically predicts higher power amplification ratio, its output level was measured lower than even that of the single channel device. This is because the magnets move slower than in the single channel configuration. It is also observed that the all connected multichannel device showed better result than the bundle system device because of the enhanced friction in the bundled-channels where dry (air) medium provides greater frictional losses.

\section{Circuitry}

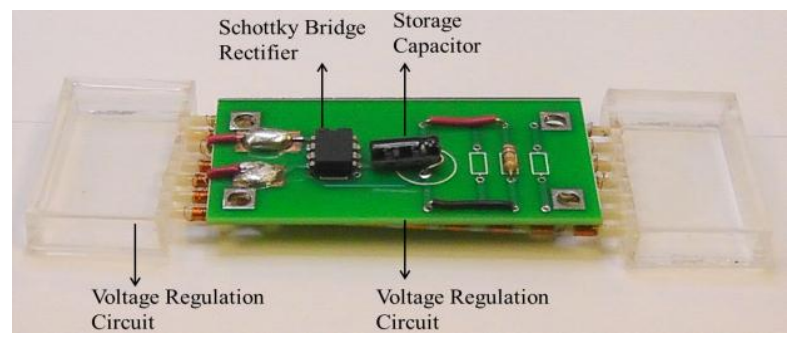

Figure 8: A: View of the 7-channel device with the voltage regulation circuit set for a shoe-embedded application.

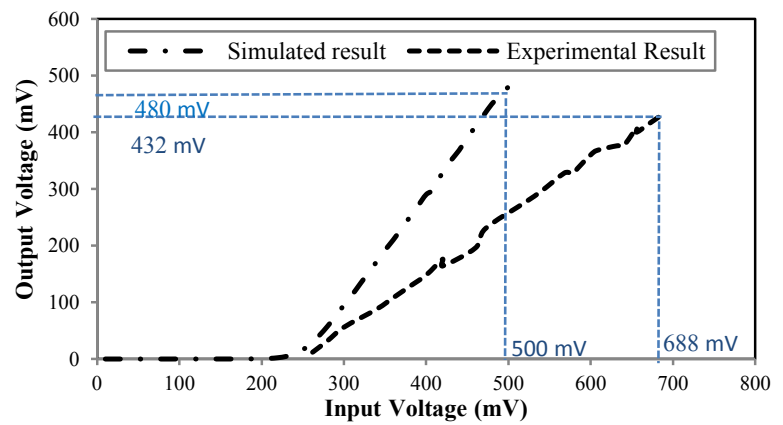

Figure 9: Simulated and experimental results for the performance of the power regulation circuit.

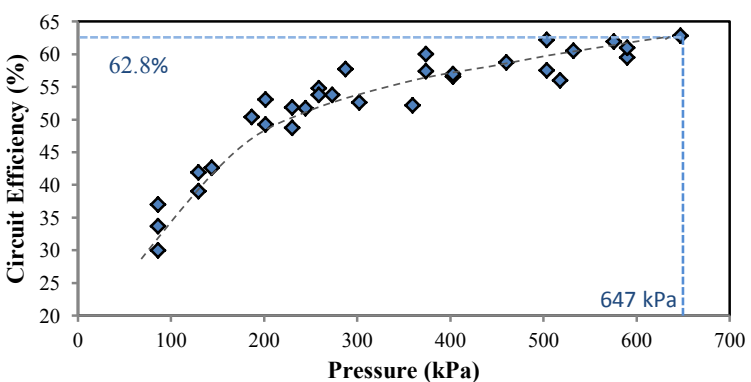

Figure 10: Graph of voltage regulation circuit efficiency verses input pressure.

The produced power was managed by the integrated hybrid circuitry that includes AC-to-DC converter as well as power storage capacity (Fig.8). The power output from each channel is a AC signal, and is first converted to DC Signal before storing in order to make the circuit more energy efficient. The AC to DC conversion is performed using an off-chip single phase Schottky Bridge Rectifier (Digi-key: 296-11231-5-ND) [12] as lesser voltage drop is seen across it. The multiple DC signals from multiple channels after conversion were added and stored across a capacitor. Figure 9 shows the experimentally tested and simulated results of the integrated circuitry with the device. The circuit efficiency (Fig. 10) increases with input pressure as the voltage level increase, giving a maximum efficiency of $62.8 \%$ with the device.

\section{CONCLUSION}

The hydraulic based energy harvesting system has been fabricated with integrated circuitry. The developed device is flexible and has a flat structure, making it convenient to be embedded into the shoe insole and power up commercial wireless devices. The two different approaches to realize a multi-channel device displayed power amplification with increase in number of channels. Further optimization of the distance between the channel for the bundle channel system is necessary. The all-connected showed greater power output with raw power ${ }_{\text {rms }}$ of $7.28 \mathrm{~mW}$ and power density of $1 \mathrm{~mW} / \mathrm{cm}^{3}$ under $647.5 \mathrm{kPa}$ input pressure. The integrated power successfully converted AC-to-DC and stored the power with $62.8 \%$ efficiency.

\section{REFERENCE}

[1] D. Noguet, G. Masera, V. Ramakrishnan, M. Belleville, D. Morche, and G. Asheid, "Considering Microelectronic Trends in Advanced Wireless System Design", Advances in electronics and telecommunications, vol. 1, no. 1, (2010).

[2] K.A. Townsend, J.W. Haslett, T.K.K. Tsang, M.N. El-Gamal and K. Iniewski, "Recent Advances and Future Trends in Low Power Wireless Systems for Medical Applications", 9th International Database Engineering \& Application Symposium, (2005).

[3] S. Basrour, B. Charlot, M. Marzencki, A. Grasso, M. Colin, and L. Valbin, "Design and fabrication of piezoelectric micro power generators for autonomous microsystems", Design, Test, Integration and Packaging of MEMS/MOEMS (2005).

[4] H Kulah, and K. Najafi "An electromagnetic micro power generator for low-frequency environmental vibrations Micro Electro Mechanical Systems", 17th IEEE Conference on MEMS, (2004).

[5] J. Yang, Fuzhi Lu, L.W. Kostiuk, and D.Y. Kwok "Electrokinetic power generation via streaming potential in microchannel: A Mobile-Ion-Drain method to increase streaming potentials", International Conference on MEMS, NANO and Smart Systems, (2004).

[6] S.H. Kim, C.H. Ji, P. Galle, and F. Herrault, " An electromagnetic energy scavenger from direct airflow" PowerMEMS, (2008).

[7] N.N.H. Ching, H. Y. Wong, W.J. Li, P.H. W. Leong, and Z. Wen, "A laser-micromachined vibrational to electrical power transducer for wireless sensing systems" Solid-State Sensors and Actuators (2001).

[8] N.S. Shenck, and J.A. Paradiso, "Energy scavenging with shoe-mounted piezoelectric" IEEE Micro. vol.21, no.3 (2001), , pp.30-42.

[9] S.R. Platt, S. Farritor, K. Garvin, and H. Haider "The use of piezoelectric ceramics for electric power generation within orthopedic implants", IEEE/ASME transactions on mechatronics, (2005).

[10] A. Bramhanand, and H. Kim, "Micro fluidic energy harvesting system for high force and large deflection accommodation", PowerMEMS, (2011).

[11] A. Bramhanand, Y.C. Bae, M.S. Kang, and H. Kim, "Prototype of a fluidics-based Energy Harvesting System", ICEIC (2012).

[12] Horowitz, Paul and W. Hill, The Art of Electronics, Second Ed., Cambridge University Press, (1989), pp. 44-47. 